\title{
Iterative Learning Control of Functional Electrical Stimulation in the Presence of Voluntary User Effort
}

\author{
Sakariya Sa-e, Christopher T. Freeman, Kai Yang \\ School of Electronics and Computer Science, University of Southampton, Southampton SO17 1BJ, UK
}

\begin{abstract}
Worldwide 17 million people are left with impairment to their upper or lower limb following stroke. Functional electrical stimulation (FES) is a method of artificially activating muscles using electrical pulses and is the most common rehabilitation technology. A significant body of clinical research confirms that successful rehabilitation requires FES to be applied in a way that supports voluntary intention during repeated attempts at functional tasks. Electromyography (EMG) measures the voluntary contraction of muscles and has been used to directly control FES in openloop, however it is limited by poor accuracy. On the other hand, model-based feedback control can provide high accuracy, but does not explicitly promote voluntary intention.

A new dynamic model of the muscle activation, generated by combined voluntary nerve signals and FES, is developed in this paper. It includes both nonlinear recruitment and linear activation dynamics. An efficient identification procedure is then formulated which can be applied to people with stroke. A model-based hybrid EMG/FES control scheme is then derived based on the model structure, allowing tracking and volitional intention support to be simultaneously optimized for the first time. Exploiting the repeated nature of rehabilitation, the control framework is then extended to further improve tracking accuracy. That is achieved by learning from experience through iterative learning control. The framework is experimentally tested with results confirming it can deliver greater performance compared to existing FES approaches, which do not consider voluntary action in the model or controller.
\end{abstract}

Keywords: Stroke Rehabilitation, Iterative Learning Control, Functional Electrical Stimulation, Electromyography

\section{Introduction}

Sixty-five million people currently live with stroke (World Stroke Organization, 2018), and two thirds have some form of impairment in upper limb function. Repeated practice of ${ }^{30}$ functional, goal-orientated tasks are required to facilitate the process of motor re-learning. Conventional therapy involving manual exercises facilitated by a therapist has not been effective [1], and has led to a growing interest in assistive technologies. These have the potential to deliver more intense, moti- ${ }^{35}$ 10 vating practice of functional upper limb tasks required for daily living. Biofeedback is a common example which typically measures muscle activity using electromyography (EMG), and has been shown to promote voluntary effort during training [2].

Functional electrical stimulation (FES) is the most prevalent 40 assistive technology [2] and involves artificially activating impaired muscles to assist task practice. It has the advantage of enabling movement and thereby facilitating feedback in the case of muscles that are highly impaired or paralysed. The effectiveness of rehabilitation using FES is supported by clinical evi- 45 dence, neurophysiology, and motor re-learning research. In particular, a meta-analysis study advocated FES [3], and strongly indicated that effectiveness improves if the assistance provided supports the patient's voluntary intention to move their impaired limb. Effective rehabilitation must therefore promote the 50 greatest voluntary contribution from the patient, which is then complemented by precisely controlled FES to complete the intended task. Model-based feedback control design of FES is hence important in delivering accurate tracking in the presence of disturbances and noise. Surprisingly, it is largely absent from clinical practice. The few existing model-based controllers used clinically capture the response of muscles to FES, but unfortunately do not embed the volitional control action associated with the patient's residual voluntary effort. Consequently, the model accuracy degrades as the patient recovers voluntary action.

Existing FES controllers which employ biofeedback do not embed dynamic models. They comprise direct feedback of EMG signals, but suffer from poor performance in accurate task completion. To the authors' knowledge, the only control structure combining both voluntary effort and a dynamic model is developed in [4, 5, 6]. Here the model takes the form of saturated gain blocks independently applied to both FES and EMG input signals with the outputs added before being applied to a linear dynamic system representing the muscle dynamics. A feedback approach called ' $\lambda$-control' is then applied. This is implemented by measuring the muscle recruitment, which is then scaled by a single gain. The gain selection requires knowledge of only a single parameter in the model. This is found via least-square fitting of experimental data. This approach therefore trades simplification of identification for overall accuracy. Note that in [5], an artificial neural network (ANN) was also 
used to model the combined FES and EMG response, but used it for prediction, rather than control.

Although no more general form of model-feedback controller has yet embedded biofeedback, other research has attempted to indirectly capture the effect of voluntary effort by using purely position data. This was first achieved in [7] using iterative learning control (ILC), a technique that uses data, col-115 lected over previous attempts at a tracking problem, to update the control input. ILC was applied to FES-assisted walking in [8, 9, 10] and has also been used to control robotic orthoses for assisted gait in [11, 12]. ILC was used to control both FES and robotic support to assist cycling in [13]. Gait and cycling naturally consist of repeated movements and ILC has performed well when tested with unimpaired subjects applying no volun-120 tary input, such as in [13]. Performance in tests with patients applying voluntary effort has resulted in greater mean errors of around 10 degrees (ankle pitch) after 6 cycles [9]. The effect of accuracy degradation due to patients' voluntary input was explicitly stated as causing poorer performance in [9].

ILC has been applied to FES-assisted upper limb movements in [14, 15, 16, 17], where the intention is to help patients perform repeated arm movements and thereby promote re-learning of lost function. Here ILC again showed high levels of tracking

75 accuracy when tested with unimpaired subjects who provided ${ }_{130}$ no voluntary effort [17]. ILC remains one of the only modelbased FES control approaches to have been clinically applied in upper limb rehabilitation. ILC was used in five clinical feasibility studies [18, 19, 20] and led to statistically significant im-

80 provements in both clinical outcome measures, and in tracking ${ }_{135}$ accuracy over the course of the intervention. However, because existing ILC schemes have neglected the patients' voluntary action, degraded tracking accuracy has been observed especially in patients with less severe stroke (e.g. in [20] the least im-

85 paired participant had the least improvement in Fugl-Meyer as-140 sessment score). In addition, existing ILC schemes also do not have the ability to promote voluntary action, which has potentially limited therapeutic outcomes to date.

The models used in all the above ILC designs do not take

${ }_{90}$ into account that motion is elicited by both FES and voluntary ${ }_{145}$ effort. It is therefore unsurprising that performance degrades when voluntary effort is omitted. Theoretically, the error can still converge to zero, but only if the voluntary effort is the same on each trial, and the dynamic relationship between voluntary

95 effort and output is linear. Unfortunately, these assumptions do 150 not generally hold.

This paper develops a novel hybrid model embedding simultaneous volitional EMG and FES induced muscle recruitment together with muscle activation dynamics. The structure is mo-

100 tivated by the underlying biophysical properties with model $_{155}$ parameters found via a clinically-relevant identification procedure. The general utility of this hybrid model is demonstrated through the development of model-based controllers that can precisely balance the relative contribution of voluntary effort and FES assistance for the first time. As a result, an ILC scheme 160 is derived which theoretically provides perfect tracking in the presence of arbitrary voluntary effort. This is the first time that an ILC scheme has combined both FES and EMG. This ILC structure differs from conventional designs since it effectively adjusts the model used to update the control input on every trial. To implement the controller, a hardware design is proposed using 'electronic blanking' to enable simultaneous EMG recording and FES application. Using this system, experimental tests are undertaken which validate the modelling and controller efficacy.

Note that preliminary results have been published in [21], however those results employed a simpler identification procedure, did not include ILC, and contained no experimental data.

\section{Hybrid FES/EMG model development}

A wide variety of models of activated muscle exist, with physiological aspects first captured in the Huxley model based on cross-bridge kinematics [22]. The earliest mechanical representation was proposed by Hill [23], which was further developed in [24, 25, 26]. More complex forms then evolved that could model the dynamic properties of limb motion in response to FES, including nonlinear coupled systems [27].

The most prevalent dynamic representation for FES control design models the dynamic response of muscle as the product of a force length, force velocity property, and muscle activation dynamics under isometric conditions. The first two forms are selected to correspond with physiological observation [28, 29, 30], but they have also been combined in a functional form [31]. The latter predominantly takes the form of a Hammerstein structure [32] incorporating a static nonlinearity called the isometric recruitment curve (IRC) in series with linear activation dynamics (LAD). The IRC has been parameterized in several ways, taking the form of saturated gain blocks [33], splines [34], and a piecewise linear function [29, 35]. The LAD has been shown to be satisfactorily modelled by a critically damped second order [36, 37], however, it is shown in [38] that higher orders provide slightly improved accuracy. It is important to note that the aforementioned models do not distinguish between voluntary effort or induced action potentials.

The biophysics that governs both natural and evoked activation of muscle is well documented, but must be embedded within a general structure that is suitable for subsequent control design. Electrical stimulation of a motor nerve produces both orthodromic and antidromic responses. The antidromic response emanates towards the spinal cord and may block any volitional action potential from the central nervous system, thereby partially eliminating volitional recruitment. This blocking action means that recruitment is a nonlinear, static function of both volitional action potential (measured by EMG), and induced action potential (elicited by FES). The characteristics of the nonlinear mapping have been confirmed experimentally in [39], and have a general form that involves monotonically increasing maps between variables.

The aforementioned biophysics motivates the structure shown in Fig. 1 to capture the dynamic interaction. Here a discrete form is assumed with signals defined over samples $i=0, \ldots, N$. For simplicity, and without loss of generality, zero initial conditions are assumed since the response to initial condition can be absorbed into the reference. This matches 


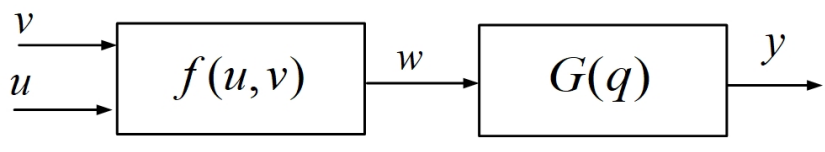

Figure 1: Hammerstein structure using hybrid activation model

most rehabilitation training because the patient's limb is moved to the same starting position between attempts. The model has the following formal definition.

Definition 1. (Muscle Model). Let $u, v, w, y$ belong to signal space $l_{2}[0, N]$. The force, $y$, generated in response to FES signal $u$ and volitional signal $v$ is given by

$$
y=G(q) f(u, v) .
$$

The LAD component is given by $y=G(q) w$ where $G(q)$ is a single-input single-output (SISO) transfer function driven by muscle recruitment $w$. The memoryless IRC operator is defined by

$$
w=f(u, v)
$$

with the naturally arising biological constraints

$$
u(i) \in\left[0, u_{\text {max }}\right] \subset \mathbb{R}, v(i) \in\left[0, v_{\text {max }}\right] \subset \mathbb{R}, w(i) \in\left[0, f_{\text {max }}\right] \subset \mathbb{R}
$$

where, as a consequence of the biophysics, the muscle recruitment increases monotonically as a function of both u or v, i.e.

$$
\frac{\partial}{\partial u} f(u, v)>0, \frac{\partial}{\partial v} f(u, v)>0
$$

with the maximum value $f_{\max }:=f\left(u_{\max }, v_{\max }\right)$.

Note: If $v=0$, the form $f(u, 0)$ equals the special case commonly used in traditional FES model-based control [16].

\subsection{Identification procedure}

There are many identification methods available for Hammerstein structures (see e.g. [34, 37, 40]), however it was shown in [41] that most methods are unsuitable for rehabilitation due to structured constraints and/or a test procedure that is not suitor use with patients. A similar approach to that of [41] is therefore adopted and expanded to the current multiple input structure.

In the clinical setting, there is minimal set-up time, reduced control over environment constaints and little possibility of retasks. This necessitates the following simple, robust identification procedure to capture the model parameters.

Definition 2. (Identification Problem). Consider the Muscle Model of Definition 1 with $G(q)$ written as $B(q) / A(q)$ and parametrized by $\theta$ and IRC $f(\cdot)$ parametrized by $\psi$. Then, given a set of data $\{\tilde{y}, \tilde{u}, \tilde{v}\}$, the standard auto regressive external noise identification problem is to find solutions $\hat{\theta}$ and $\hat{\psi}$ such that

$$
(\hat{\theta}, \hat{\psi})=\underset{\theta, \psi}{\operatorname{argmin}}\|A(q, \theta) \tilde{y}-B(q, \theta) f(\tilde{u}, \tilde{v}, \psi)\|_{2} .
$$

As stated, the nonlinearity can be modelled by splines, saturated gains, or piecewise linear segments. Here the latter has been adopted. This leads to the following parametric structures.
Definition 3. (IRC and LAD Parametric Forms). Let $f(u, v)$ be a piecewise linear function with independent vertex coordinates $\left\{\bar{u}_{i}\right\}_{i=1 \cdots n},\left\{\bar{v}_{j}\right\}_{j=1 \cdots m}$. The associated parameters are $\left\{f\left(\bar{u}_{i}, \bar{v}_{j}\right)\right\}_{i, j}$ which are arranged in the vector form

$$
\psi=\left[\begin{array}{llllll}
f\left(\bar{u}_{1}, \bar{v}_{1}\right) & \cdots & f\left(\bar{u}_{1}, \bar{v}_{m}\right) & f\left(\bar{u}_{2}, \bar{v}_{1}\right) & \cdots & f\left(\bar{u}_{n}, \bar{v}_{m}\right)
\end{array}\right]^{T} .
$$

Similarly, let the linear dynamics have form

$$
G(q)=\frac{\beta_{0} q^{-\tau}+\beta_{1} q^{-(\tau+1)}+\cdots+\beta_{b} q^{-(\tau+b)}}{1+\alpha_{1} q^{-1}+\cdots+\alpha_{a} q^{-a}},
$$

where $q^{-1}$ is the delay operator, and $a, b$ and $\tau$ are the number of poles, zeros and the time delay order respectively, then

$$
\theta=\left[\begin{array}{ll}
\theta_{\alpha} & \theta_{\beta}
\end{array}\right]^{T}, \theta_{\alpha}=\left[\begin{array}{lll}
\alpha_{1} & \cdots & \alpha_{a}
\end{array}\right], \theta_{\beta}=\left[\begin{array}{lll}
\beta_{0} & \cdots & \beta_{b}
\end{array}\right]
$$

are the parameters of the $L A D$ component. Note that $a, b$ and $\tau$ are fixed in advance for each identification, but it can be repeated for difference values.

Theorem 1. Given a fixed estimate $\hat{\psi}$ of parameter $\psi$ in Definition 2 an optimal estimate of the LAD parameter is

$$
\hat{\theta}=\left(\Theta(\tilde{y}, f(\tilde{u}, \tilde{v}, \hat{\psi}))^{T} \Theta(\tilde{y}, f(\tilde{u}, \tilde{v}, \hat{\psi}))\right)^{-1} \Theta(\tilde{y}, f(\tilde{u}, \tilde{v}, \hat{\psi}))^{T} y
$$

where $\Theta=$

$\left[\begin{array}{cccccc}-\tilde{y}(a) & \cdots & -\tilde{y}(1) & f(\tilde{u}, \tilde{v}, \hat{\psi})(a+1-\tau) & \cdots & f(\tilde{u}, \tilde{v}, \hat{\psi})(a+1-\tau-b) \\ -\tilde{y}(a+1) & \cdots & -\tilde{y}(2) & f(\tilde{u}, \tilde{v}, \hat{\psi})(a+2-\tau) & \cdots & f(\tilde{u}, \tilde{v}, \hat{\psi})(a+2-\tau-b) \\ \vdots & & \vdots & \vdots & & \vdots \\ -\tilde{y}(N-1) & \cdots & -\tilde{y}(N-a) & f(\tilde{u}, \tilde{v}, \hat{\psi})(N-\tau) & \cdots & f(\tilde{u}, \tilde{v}, \hat{\psi})(N-\tau-b)\end{array}\right]$

and

$$
y=\left[\begin{array}{llll}
\tilde{y}(a+1) & \tilde{y}(a+2) & \cdots & \tilde{y}(N)
\end{array}\right]^{T} .
$$

Given a fixed estimate $\hat{\theta}$ of parameter $\theta$ in Definition 2. if constraint (4) is inactive, an optimal estimate of the IRC parameter is

$$
\hat{\psi}=\left(\left(X\left(\hat{\theta}_{\beta}\right) \Phi(\tilde{u}, \tilde{v})\right)^{T}\left(X\left(\hat{\theta}_{\beta}\right) \Phi(\tilde{u}, \tilde{v})\right)\right)^{-1}\left(X\left(\hat{\theta}_{\beta}\right) \Phi(\tilde{u}, \tilde{v})\right)^{T} Y\left(\tilde{y}, \hat{\theta}_{\alpha}\right)
$$

where for $l=1, \cdots, N$, matrix $\Phi(\tilde{u}, \tilde{v})$ has elements

$$
\begin{aligned}
\Phi_{l,(i \cdot n)+1} & =\frac{\tilde{u}_{(l)}-\bar{u}_{i}}{\bar{u}_{i+1}-\bar{u}_{i}} s(l, i, j), \\
\Phi_{l,(i-1) n+j+1} & =\frac{\tilde{v}_{(l)}-\bar{v}_{j}}{\bar{v}_{j+1}-\bar{v}_{j}} s(l, i, j), \\
\Phi_{l,(i \cdot n)+j-(s(l, i, j)+1)_{(n+1) / 2}} & =1-\Phi_{l,(i \cdot n)+1}-\Phi_{l,(i-1) n+j+1}
\end{aligned}
$$

where $i=\max \left\{p \mid \bar{u}_{p}<\tilde{u}_{(l)}\right\}, j=\max \left\{p \mid \bar{v}_{p}<\tilde{v}_{(l)}\right\}$ and $s(l, i, j)=\operatorname{sgn}\left(\left(\tilde{v}_{(l)}-\bar{v}_{j+1}\right)-\left(\tilde{u}_{(l)}-\bar{u}_{i}\right) \bar{u}_{i}-\bar{v}_{i+1}-\bar{v}_{j}\right)$. All other of elements $\Phi(\tilde{u}, \tilde{v})$ are zero. Also

$$
Y\left(\tilde{y}, \hat{\theta}_{\alpha}\right)=\left[\begin{array}{c}
\tilde{y}(a+1)+\alpha_{1} \tilde{y}(a)+\cdots+\alpha_{a} \tilde{y}(1) \\
\tilde{y}(a+2)+\alpha_{1} \tilde{y}(a+1)+\cdots+\alpha_{a} \tilde{y}(2) \\
\vdots \\
\tilde{y}(N)+\alpha_{1} \tilde{y}(N-1)+\cdots+\alpha_{a} \tilde{y}(N-a)
\end{array}\right],
$$




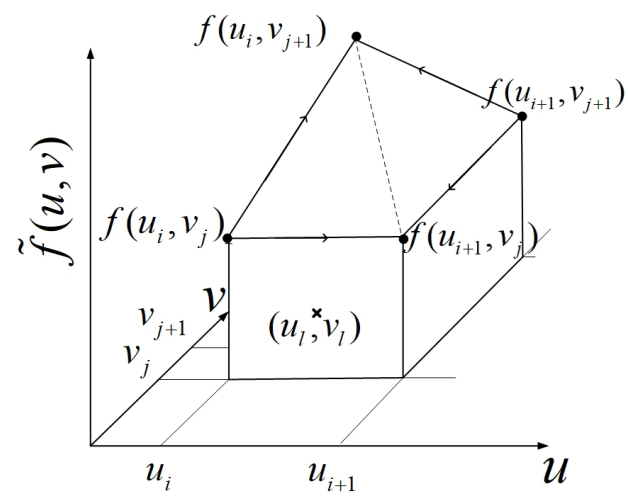

Figure 2: Points used to define piecewise function $f(u, v)$

and

$$
X\left(\hat{\theta}_{\beta}\right)=\left[\begin{array}{ccccccc}
\beta_{a-\tau} & \beta_{a-\tau-1} & \cdots & \beta_{0} & 0 & \cdots & 0 \\
0 & \beta_{a-\tau} & \cdots & \beta_{1} & \beta_{0} & \cdots & 0 \\
\vdots & \vdots & \ddots & \vdots & \vdots & \ddots & 0 \\
0 & 0 & \cdots & \beta_{a-\tau} & \beta_{a-\tau-1} & \cdots & \beta_{0}
\end{array}\right]
$$

If constraint (4) is active, solution (9) is replaced by

$$
\hat{\psi}:=\min _{\psi}\left\|Y\left(\tilde{y}, \hat{\theta}_{\alpha}\right)-\left(X\left(\hat{\theta}_{\beta}\right) \Phi(\tilde{u}, \tilde{v})\right) \psi\right\|_{2} \text {, such that } \psi<0
$$

which can be solved using any suitable numerical algorithm (e.g. the interior-point method).

Proof: Write $G(q)=B(q) / A(q)$ and express (5) in form

$$
\hat{\theta}=\underset{\theta}{\operatorname{argmin}}\|A(q) y-B(q) f(\tilde{u}, \tilde{v}, \hat{\psi})\|_{2}
$$

which can be written in matrix form as

$$
\underset{\theta}{\operatorname{argmin}}\|y-\Theta(\tilde{y}, f(\tilde{u}, \tilde{v}, \hat{\psi})) \theta\|_{2}
$$

with solution

$$
\hat{\theta}=\Theta^{\dagger} y,
$$

where $\dagger$ denotes the pseudoinverse. The IRC identification similarly becomes

$$
\hat{\psi}=\underset{\psi}{\operatorname{argmin}}\|A(q) \tilde{y}-B(q) f(\tilde{u}, \tilde{v}, \psi)\|_{2} .
$$

Consider $f(\cdot)$ acting on a data point $\left(\tilde{u}_{l}, \tilde{v}_{l}\right)$ shown in Fig. 2 If

$$
\tilde{v}_{l}-\tilde{v}_{j+1} \leq\left(\tilde{u}_{l}-u_{i}\right) \frac{u_{i}-u_{i+1}}{v_{j+1}-v_{j}}
$$

then $f\left(\tilde{u}_{l}, \tilde{v}_{l}\right)$ lies in the plane containing points

$$
\left[\begin{array}{lll}
f\left(\bar{u}_{i}, \bar{v}_{j}\right) & f\left(\bar{u}_{i}, \bar{v}_{j+1}\right) \quad f\left(\bar{u}_{i+1}, \bar{v}_{j}\right)
\end{array}\right]^{T} .
$$

Otherwise it lies in plane containing points

$$
\left[f\left(\bar{u}_{i+1}, \bar{v}_{j+1}\right) \quad f\left(\bar{u}_{i}, \bar{v}_{j+1}\right) \quad f\left(\bar{u}_{i+1}, \bar{v}_{j}\right)\right]^{T}
$$

In the first case, the point $f\left(\tilde{u}_{l}, \tilde{v}_{l}\right)$ is given by

$f\left(\tilde{u}_{(l)}, \tilde{v}_{(l)}\right)=\left[\begin{array}{lll}\left(1-\frac{\tilde{u}_{(l)}-\bar{u}_{i}}{\bar{u}_{i+1}-\bar{u}_{i}}-\frac{\tilde{v}_{(l)}-\bar{v}_{j}}{\bar{v}_{j+1}-\bar{v}_{j}}\right) & \frac{\tilde{v}_{(l)}-\bar{v}_{j}}{\bar{v}_{j+1}-\bar{v}_{j}} & \tilde{u}_{(l)}-\bar{u}_{i} \\ \bar{u}_{i+1}-\bar{u}_{i}\end{array}\right]\left[\begin{array}{c}f\left(\bar{u}_{i}, \bar{v}_{j}\right) \\ f\left(\bar{u}_{i}, \bar{v}_{j+1}^{2+1}\right) \\ f\left(\bar{u}_{i+1}, \bar{v}_{j}\right)\end{array}\right]$.
In the second case, the point $f\left(\tilde{u}_{l}, \tilde{v}_{l}\right)$ is given by

$$
f\left(\tilde{u}_{(l)}, \tilde{v}_{(l)}\right)=\left[\left(1+\frac{\tilde{u}_{(l)}-\bar{u}_{i}}{\bar{u}_{i+1}-\bar{u}_{i}}+\frac{\tilde{v}_{(l)}-\bar{v}_{j}}{\bar{v}_{j+1}-\bar{v}_{j}}\right)-\frac{\tilde{v}_{(l)}-\bar{v}_{i}}{\bar{v}_{i+1}-\bar{v}_{i}}-\frac{\tilde{u}_{(l)}-\bar{u}_{j}}{\bar{u}_{j+1}-\bar{u}_{j}}\right]\left[\begin{array}{c}
f\left(\bar{u}_{i+1}, \bar{v}_{j+1}\right) \\
f\left(\bar{u}_{i}, \bar{v}_{j+1}\right) \\
f\left(\bar{u}_{i+1}, \bar{v}_{j}\right)
\end{array}\right] .
$$

These definitions correspond to the form $f(\tilde{u}, \tilde{v})=\Phi(\tilde{u}, \tilde{v}) \psi$ with element (10) and the problem (12) can be expressed in lifted form as

$$
\underset{\psi}{\operatorname{argmin}}\left\|Y\left(\tilde{y}, \hat{\theta}_{\alpha}\right)-\left(X\left(\hat{\theta}_{\beta}\right) \Phi(\tilde{u}, \tilde{v})\right) \psi\right\|_{2}
$$

where $\Phi$ contains the elements of $\Phi_{l,(i, j)}$. The solution of 13 is

$$
\hat{\psi}=\left(X\left(\hat{\theta}_{\beta}\right) \Phi(\tilde{u}, \tilde{v})\right)^{\dagger} Y\left(\tilde{y}, \hat{\theta}_{\alpha}\right)
$$

Remark 1. The minimisation (5) over $\hat{\theta}, \hat{\psi}$ parameters can be executed iteratively by computing solution (7) and (9) (or (11)) in an alternating fashion and stopping when the change in the cost function is below a user-defined bound. A similar iterative approach was used for artificially stimulated muscle identification in [41, 42] and worked effectively.

\subsection{Test Procedure}

Theorem 1 and Remark 1 require test data $\{\tilde{u}, \tilde{v}, \tilde{y}\}$ that sufficiently excite both IRC and LAD model components. An efficient method to generate such data is by using two separate tests: one to provide data for use in the IRC identification (11) and the other for use in the LAD identification (7). Algorithms 1 and 2 provide efficient test procedures for this purpose.

Algorithm 1 specifies that a set of stimulation intensities $\left\{u_{i}\right\}$ is applied to the participant who is initally relaxed, but then gradually increases their volitional force until a maximum force level is attained. The output responses, $\left\{y_{i}\right\}$, stimulation intensities, $\left\{u_{i}\right\}$, and voluntary EMG, $\left\{v_{i}\right\}$, are recorded using specialist hardware designed for simultaneously applying FES and recording EMG. This hardware is described in Section 3.1

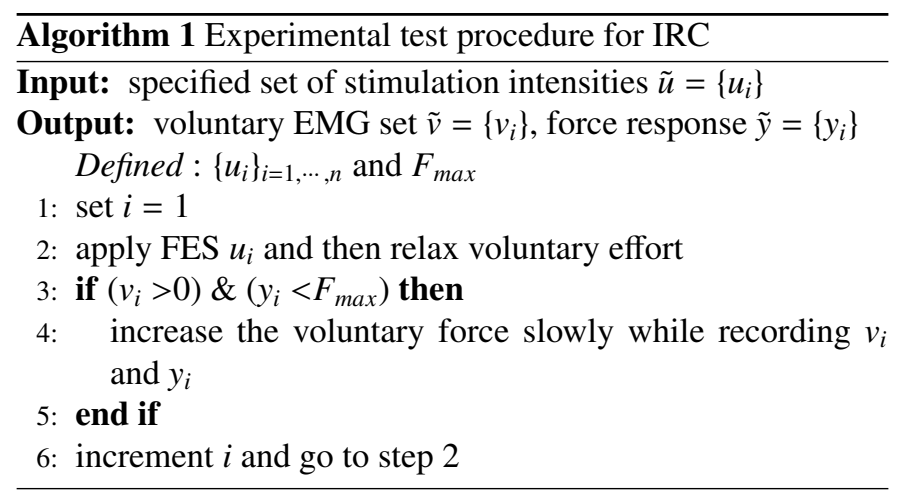

Algorithm 2 measures output responses, $\left\{y_{i}\right\}$ when sufficiently exciting stimulation signals $\left\{u_{i}\right\}$ are applied to the participant with no voluntary effort.

The procedure of Remark 1 is then applied to solve the identification problem of Definition 2 


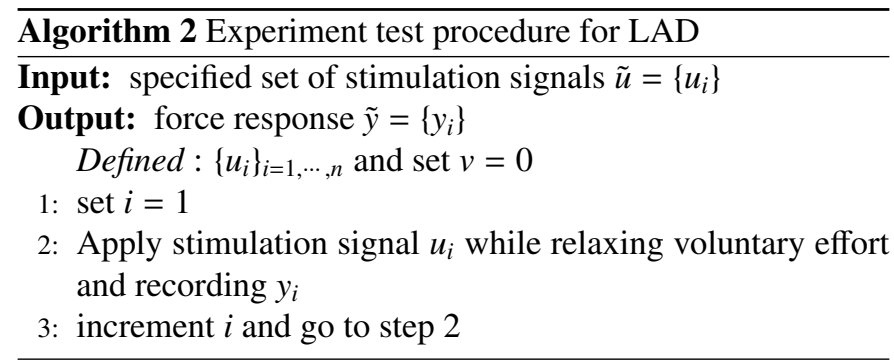

\subsection{Optimal ILC Design}

As previously highlighted, existing controllers using voluntary effort and FES do not embed a model. They either amplify voluntary effort (measured by EMG), or compensate for fatigue (as in [4]) and hence do not explicitly assist tracking of a reference. The current focus is to accurately support task tracking for use in rehabilitation, which necessitates controlling the stimulation signal in order to balance encouragement of voluntary contribution and achieving the tracking task. Mathematically, this can be written as the optimisation problem;

$\operatorname{argmin} J(u), J(u)=\sum_{i=0}^{N}(r(i)-G(q) f(u, v)(i))^{2} Q+(f(u, v)(i))^{2} R$

where $Q$ and $R$ are positive definite weights which penalise the tracking error and the muscle recruitment signals respectively. ${ }^{245}$

This is a form of linear quadratic tracking (LQT) problem that can be solved by either feedforward or feedback control. The feedforward solution computes $u$ in advance, while the feedback control solution employs state-estimation and statefeedback. Unfortunately, both forms of LQT cannot enforce perfect tracking since they are susceptible to model uncertainty and disturbance when they are used with large $Q$ values. Therefore the problem description will be generalized by adding an update term that corrects the input after each attempt. This results in an ILC problem.

ILC is an approach used for high performance tracking of systems that repeatedly perform the same tracking task with resetting to identical initial conditions between attempts. Each attempt is called a trial and the objective is to update the control input between trials to reduce the error on the next trial. Let $k$ denotes the trial number with error $e_{k}=r-y_{k}$ where $r$ is the reference. A general ILC update law is given by

$$
u_{k+1}=u_{k}+\gamma\left(e_{k}\right)
$$

where $u_{k}$ is the stimulation input on the $k^{\text {th }}$ trial and the correction, $\gamma$, is some function of the output tracking error, $e_{k}$. The input initial conditions are assumed zero, $u_{0}=0$. The ILC problem generalises the LQT problem, $(15)$ and (since $u_{0}=v_{0}=0$ ) coincides with it when $k=0$. The first trial of ILC therefore equates to LQT. The ILC problem is expressed as follows.

Definition 4. (Hybrid ILC Problem). Consider the system $y_{k}=$ $G(q) f\left(u_{k}, v_{k}\right)$ with operators as in Definition 1 . The problem is to design FES signal $u_{k+1}$, such that tracking and trial-to-trial change in muscle recruitment are balanced on each trial, i.e. to solve

$$
\begin{aligned}
u_{k+1}:= & \operatorname{argmin} J(u), \\
J(u)= & \sum_{i=0}^{N}\left\{\left(r(i)-G(q) f\left(u, v_{k+1}\right)(i)\right)^{2} Q\right. \\
& \left.+\left(f\left(u, v_{k+1}\right)(i)-f\left(u_{k}, v_{k}\right)(i)\right)^{2} R\right\} .
\end{aligned}
$$

The output must converge to the reference as the trial number increases, i.e.

$$
\lim _{k \rightarrow \infty} y_{k}=r
$$

and the muscle recruitment must converge to a fixed signal, i.e.

$$
\lim _{k \rightarrow \infty} f\left(u_{k}, v_{k}\right)=f_{\infty}
$$

Moreover, if voluntary input $v_{k}$ converges to a fixed signal, then the applied FES $u_{k}$ must similarly converge.

The control problem in (16) is a variation of 'Norm-optimal ILC' (NOILC). This approach has received significant attention in the ILC community, and has been successful in a range of applications [18, 43, 44]. By including both tracking accuracy and a function of control effort in its cost function, NOILC avoids inverting the plant operator. Instead it uses a stable operator within its update to enable smooth convergence of the control and error signals over several trials. Weights $Q$ and $R$ provide a transparent method of varying the convergence speed. Only when $Q \rightarrow \infty$ does plant inversion occur. The solution to the hybrid ILC problem is now given.

Theorem 2. Suppose on trial $k+1$ there exists voluntary action $v_{k+1} \geq 0$ and a reference $r \geq 0$ is selected such that the muscle recruitment satisfies

$$
f\left(0, v_{k+1}\right) \leq \sum_{i=0}^{k}(I-L G)^{i} L r \leq f\left(u_{\max }, v_{k+1}\right)
$$

where $L=\left(\bar{R}+G^{T} \bar{Q} G\right)^{-1} G^{T} \bar{Q}$, in which $\bar{Q}=\operatorname{diag}\{Q, \cdots, Q\}$, and $\bar{R}=\operatorname{diag}\{R, \cdots, R\}$. Then the Hybrid ILC Problem of Definition 4 is solved by the ILC update algorithm

$$
u_{k+1}=f^{-1}\left(p_{k}+L\left(r-y_{k}\right), v_{k+1}\right)
$$

combined with

$$
p_{k+1}=p_{k}+L\left(r-y_{k}\right)
$$

where $p_{0}=0$ and $f^{-1}(\cdot)$ is an inverse operator with respect to its second argument (e.g. $\left.f\left(f^{-1}\left(p_{k+1}, v_{k+1}\right), v_{k+1}\right)=p_{k+1}\right)$. Alternatively, 20) and 21, may be replaced by

$$
u_{k+1}=f^{-1}\left(f\left(u_{k}, v_{k}\right)+L\left(r-y_{k}\right), v_{k+1}\right)
$$

in which

$$
f\left(u_{k+1}, v_{k+1}\right)=f\left(u_{k}, v_{k}\right)+L\left(r-y_{k}\right) .
$$

Proof: Cost function (16) is minimised by the solution

$$
u_{k+1}=f^{-1}\left(p_{k}+L\left(r-G(q) f\left(u_{k}, v_{k}\right)\right), v_{k+1}\right) .
$$


Plant

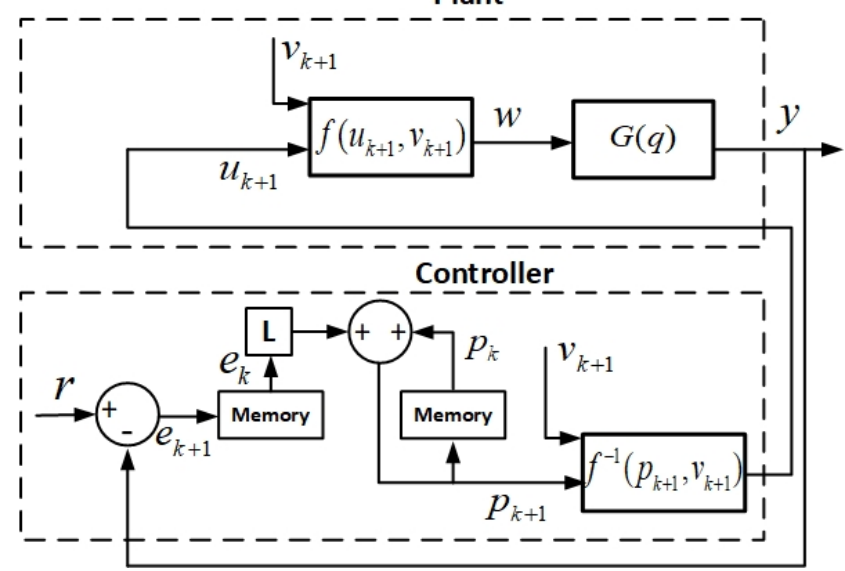

Figure 3: Feedforward ILC control structure of Theorem 2

which equates to the combined updates 20, and 21, or 22, 23. However this is only realised if $r$ and $u_{k+1}$ satisfy

$$
y_{k+1}(i) \leq r(i) \text { and } u_{k+1}(i) \leq u_{\max }
$$

Note that, if the controller did not use voluntary action information, then non-zero error would occur due to the inexact cancellation

$$
\begin{gathered}
f\left(f^{-1}\left(p_{k+1}, 0\right), v_{k+1}\right) \neq p_{k+1} \\
f^{-1}\left(f\left(u_{k+1}, v_{k+1}\right), 0\right) \geq f^{-1}\left(f\left(u_{k+1}, v_{k+1}\right), v_{k+1}\right) .
\end{gathered}
$$

The feedforward ILC control structure is shown in Fig. 3. The next remark shows that the solution to the LQT problem (15) is implemented by the first trial of ILC.

Remark 2. If $k=0$, ILC updates (20) and 21) collapse to the optimal feedforward form (standard LQT feedforward solution)

$$
u=f^{-1}\left(L\left(r-y_{0}\right), v\right)
$$

which, since $y_{0}=0$, equals

$$
u=f^{-1}\left(\left(\bar{R}+G^{T} \bar{Q} G\right)^{-1} G^{T} \bar{Q} r, v\right) .
$$

The solutions (20), (21) are of a feedforward type, and therefore do not embed error recorded in the current trial. The next result provides an alternative solution that includes current trial feedback.

Theorem 3. Theorem 2 holds with updates (20), (21) replaced by the ILC feedback form

$$
u_{k+1}=f^{-1}\left(p_{k+1}, v_{k+1}\right)
$$

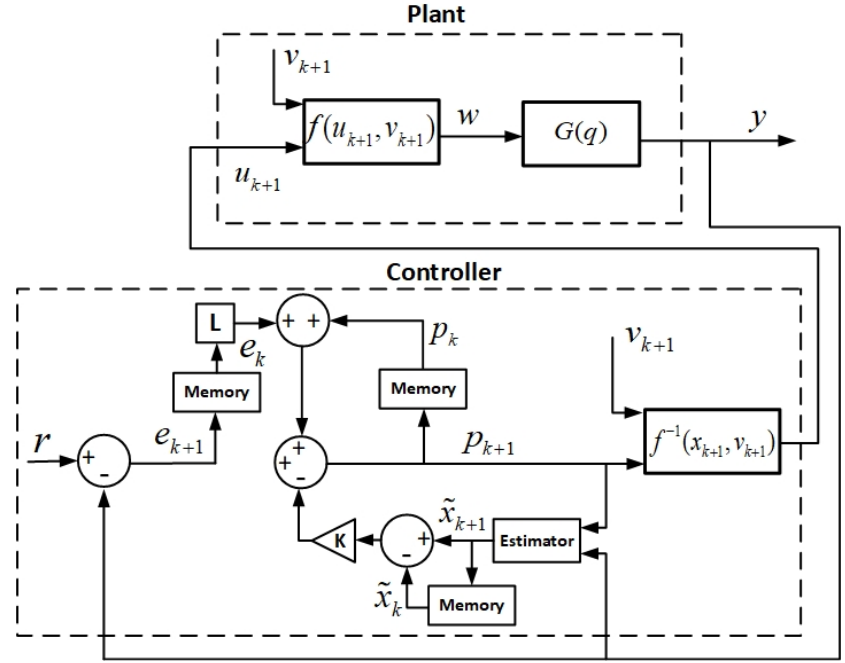

Figure 4: Feedback and feedforward ILC control structure of Theorem 3

where

$$
\begin{aligned}
p_{k+1}(i)= & p_{k}(i)+\bar{R}^{-1} B^{T} \zeta_{k+1}(i)- \\
& \left(B^{T} K(i) B\right)^{-1} B^{T} K(i) A\left(\tilde{x}_{k+1}(i)-\tilde{x}_{k}(i)\right), \\
K(i)= & A^{T} K(i+1) A+C^{T} Q C- \\
& \left(A^{T} K(i+1) B\left(B^{T} K(i+1) B+R\right)^{-1} B^{T} K(i+1) A\right), \\
\zeta_{k+1}(i)= & \left(I+K(i) B R^{-1} B^{T}\right)^{-1}\left\{A^{T} \zeta_{k+1}(i+1)\right. \\
& \left.+C^{T} Q\left(r(i+1)-y_{k}(i+1)\right)\right\}
\end{aligned}
$$

with terminal conditions $\zeta_{k}(N)=0$ and $K(N)=0$. Here $\tilde{x}_{k+1}(i)$, $\tilde{x}_{k}(i)$ are the current and previous state of the LAD system respectively.

Proof: This follows from [45] and the proof of Theorem 2,

The feedback ILC control structure is illustrated in Fig. 4.

Remark 3. If $k=0$, ILC update (26) collapses to the optimal state feedback form (standard LQT feedback solution)

$$
u(i)=f^{-1}\left(\bar{R}^{-1} B^{T} \zeta(i)-\left(B^{T} K(i) B\right)^{-1} B^{T} K(i) A \tilde{x}(i), v(i)\right)
$$

where

$$
\begin{aligned}
K(i)= & A^{T} K(i+1) A+C^{T} Q C- \\
& A^{T} K(i+1) B\left(B^{T} K(i+1) B+R\right)^{-1} B^{T} K(i+1) A, \\
\zeta(i)= & \left(I+K(i) B R^{-1} B^{T}\right)^{-1}\left\{A^{T} \zeta(i+1)+C^{T} Q r(i+1)\right\}
\end{aligned}
$$

with terminal conditions $\zeta(N)=0$ and $K(N)=0$.

\section{Identification Results}

This section applies the identification and control design approaches of Section 2 experimentally. Four healthy participants (P1 to $\mathrm{P} 4)$ were recruited to the study, following Ethics protocol 


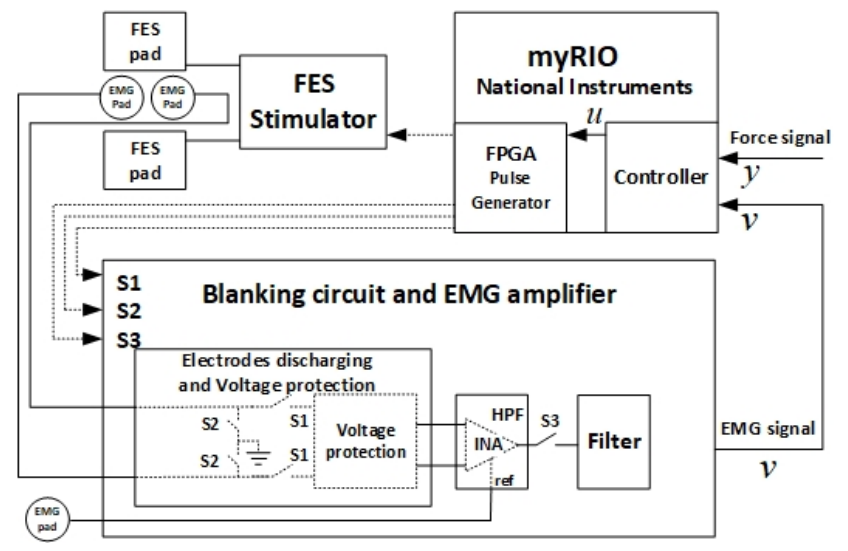

Figure 5: Hardware blanking circuit with switches S1, S2, S3, high pass filter (HPF), and field programmable gate array (FPGA)

ERGO/FEPS/47701. Each attended a single session in which the model of Section 2.1 was identified using the procedures of Section 2.2 and the control approach of Section 2.3 was then implemented and evaluated.

\subsection{Overview of Hardware}

An FES signal comprises a train of high voltage pulses with fixed period, while an EMG signal is continuous and several orders of magnitude smaller. When an EMG signal is recorded from the same muscle as FES is applied, distortion will occur to electrical interference. This distortion consists of a stimulation artefact and an M-wave [46]. To eliminate these, a hardware blanking circuit was designed, as illustrated in Fig. 5.

This circuit uses three sets of photo metal oxide semiconductor (MOS) relays activated to separate the volitional EMG from the distorted signals. The first set of switches acts at $5 m s^{320}$ in order to eliminate the stimulation artefact. The second set of switches then reduces the M-wave and are set active for 20 $m s$. The last set connects the EMG differential amplifier for 25 $m s$. These processes were applied in the same way for every riod of $50 \mathrm{~ms}$ duration, of which half this duration was em-325 ployed for recording the EMG signals. The EMG processing includes a bandpass filter with a bandwidth between 150 and $700 \mathrm{~Hz}$. For FES stimulation, a four channel stimulator (Odstock Medical Ltd., UK) was employed to deliver the electrical stimulation to the muscle. A frequency of $20 \mathrm{~Hz}$ and a com-330 fortable fixed amplitude were used for identification of a hybrid activation model. This design is an extension of initial work in [46].

The final component shown in Fig. 5 is a myRIO (National

295 Instruments, US) embedded hardware device. This has a realtime microcontroller which communicates with a field programmable gate array (FPGA) module. The control schemes (Fig. 3 and Fig. 4) are implemented on the real-time controller. The controller reads the EMG signal $v$ and force signal $y$ and computes the pulsewidth demand $u$. The latter is sent to the FPGA module which generates the required PWM signal. The FPGA module also generates the pulse signals used by the blanking circuit.

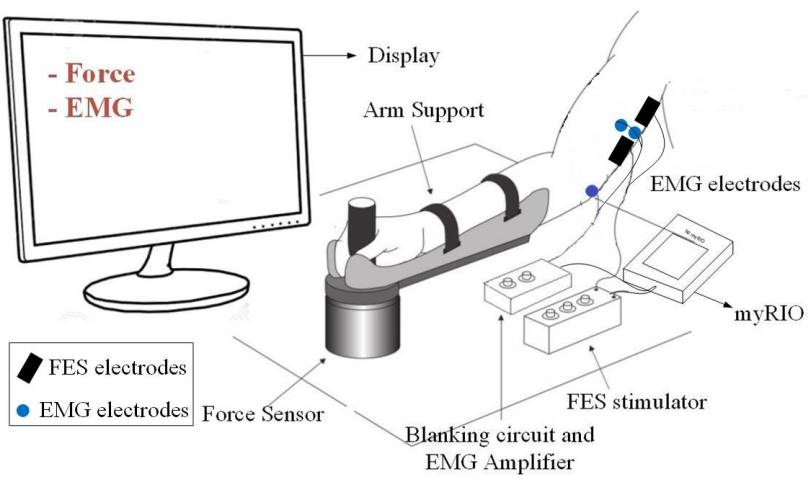

Figure 6: Experimental apparatus and electrode placement

A sampling frequency of $2000 \mathrm{~Hz}$ was employed for collecting both EMG and force data. PWM signals were applied to control the stimulation pulsewidth and the switches of the hardware blanking circuit. The force sensor is a 6 axis JR3-4604 (JR3, US).

\subsection{Test Procedure}

While the hardware, identification and controllers in this paper can be applied to any muscle, the experimental set-up and electrode arrangement are evaluated on the upper limb as shown in Fig. 6. This shows the force sensor, arm support, EMG amplifier, and stimulator. This set-up corresponds to training the triceps muscle to assist stroke patients who typically have weak extensor muscles. The aim is to track a target that corresponds to natural elbow extension as accurately as possible.

Two types of electrodes, FES electrode pads and EMG electrode pads, were used. The two FES electrode pads $(5 \times 5 \mathrm{~cm})$ were placed on the muscle belly of the triceps with on an interelectrode space of $2 \mathrm{~cm}$. Three AgCl EMG electrode pads (1.5 $\mathrm{cm}$ diameter) were used for acquiring the EMG signal, two placed between the FES electrode pads across the muscle fibre and the third pad placed on the elbow as the reference electrode.

First the test procedure of Section 2.2 was applied. This involved using Algorithm 1 with $u_{i=\{1, \cdots, 23\}}=\{80,90, \cdots, 300\} \mu \mathrm{s}$ (pulsewidth) and a maximum force of $F_{\max }=20 \mathrm{~N}$. Each test comprised voluntary effort being gradually applied to the muscle to achieve the required maximum force.

Then Algorithm 2 was applied with $\left\{u_{i}\right\}$ selected as triangular ramp functions since these have been found to be both comfortable and sufficiently exciting [17, 37, 47]. The value of $u_{\max }=300 \mu s$ was employed in each case. The force, FES, and EMG were recorded in all tests.

Then the IRC parametric forms were specified within Definition 3 , with equally spread vertices selected as

$$
\begin{aligned}
& \left\{\bar{u}_{i}\right\}=\left\{u_{\text {min }}+0 \Delta u, u_{\text {min }}+1 \Delta u, \cdots, u_{\text {min }}+(n-1) \Delta u\right\}, \\
& \left\{\bar{v}_{i}\right\}=\left\{v_{\text {min }}+0 \Delta v, v_{\text {min }}+1 \Delta v, \cdots, v_{\text {min }}+(m-1) \Delta v\right\}
\end{aligned}
$$

in which

$$
\Delta u=\frac{u_{\max }-u_{\min }}{n}, \Delta v=\frac{v_{\max }-v_{\min }}{m}
$$




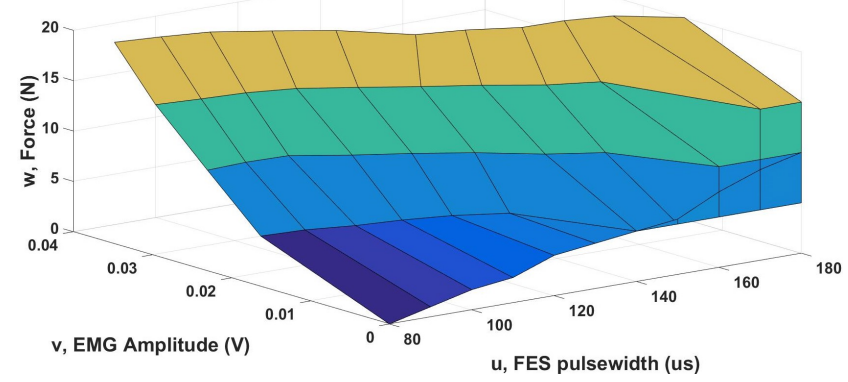

Figure 7: The hybrid activation model in raw data, $\{u, v, w\}$

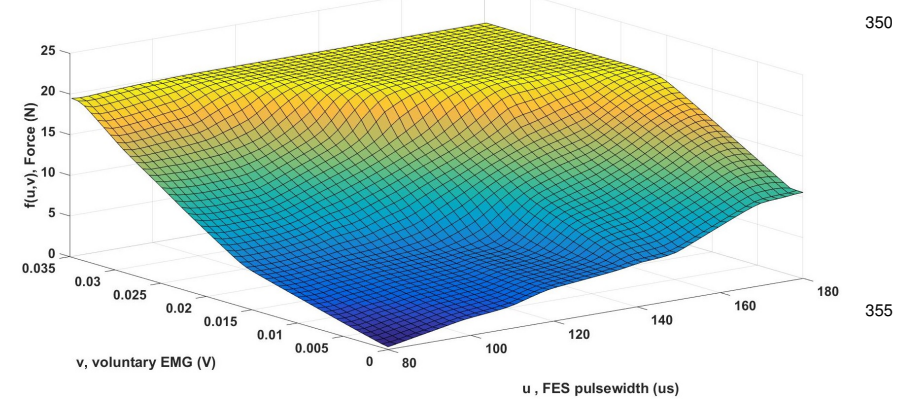

Figure 8: Identified hydrid model activation, $f(u, v)$

where $n$ and $m$ are the vertice numbers of the $u$ and $v$ axes respectively.

Having obtained the required test data $\{\tilde{u}, \tilde{v}, \tilde{y}\}$, the parameters of the model were identified through application of Remark 11. This required computing solutions (7) and (9) to respectively solve the LAD and IRC problems defined in Theorem 1

\subsection{Results}

Representative results for first participant (P1) are provided to illustrate the experimental outcomes. The raw data collected ${ }^{370}$ using Algorithm 1 for P1 is shown in Fig. 7. Applying Remark 1 then yields the optimal solution for $f(u, v)$ shown in Fig. 8 From this, the function $f^{-1}(w, v)$ required in the controller structures can be calculated, and is shown in Fig. 9 .

The results shown in Fig. 8 and 9 employ the interior-point method, implemented in Matlab using 'fmincon' with default

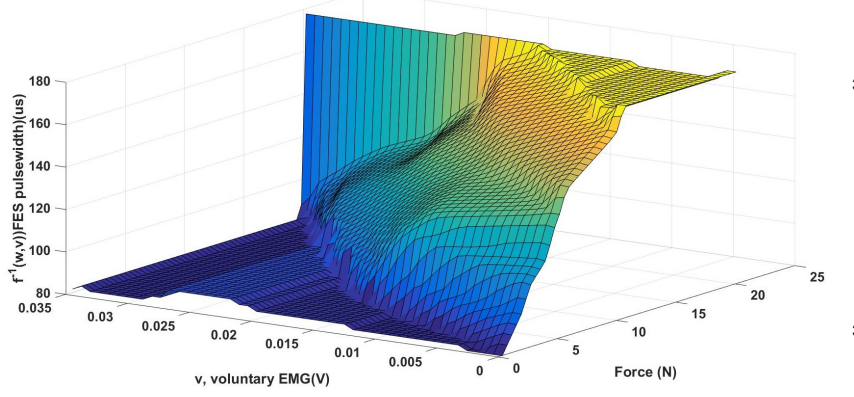

380

Figure 9: The inverse model, $f^{-1}(w, v)$

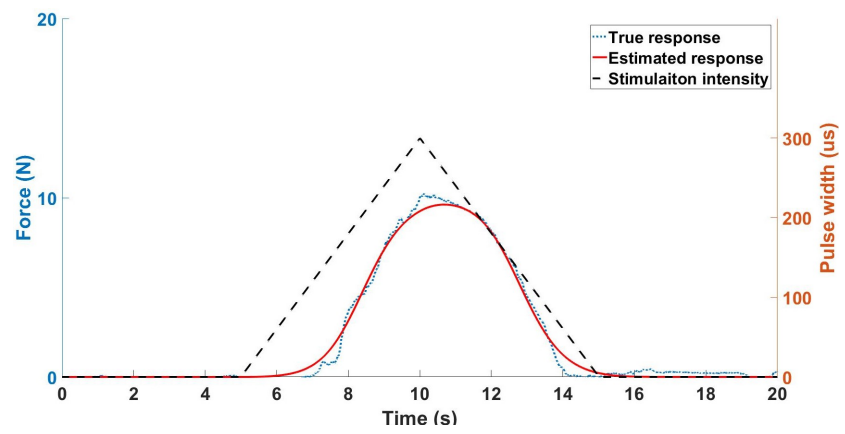

Figure 10: The LAD response

parameters. The identified LAD results for P1 are also computed and shown in Fig. 10

\section{Control Results}

The models $G(\hat{\theta}), f(u, v, \hat{\psi})$ identified in Section 3 were next used in the control designs by setting $G=G(\hat{\theta})$ and $f(u, v)=f(u, v, \hat{\psi})$. The new model-based controllers with and without ILC were implemented, in both feedforward and feedback structures. The tests were then repeated for the traditional control structures which do not include voluntary effort (i.e. the controllers were fed with $v=0$ ). The new structures are termed 'hybrid activation' (HA) controllers, and the traditional controllers are termed 'neglecting voluntary activation' (NV) controllers.

\subsection{Test procedure}

In the experimental set-up of Fig. 6, the reference and voluntary effort are shown to the participant on a display. Their arm is moved to the same starting position for each trial. The participant was instructed to provide no voluntary effort in tests which did not require voluntary assistance, and their vEMG signal was used to ensure this occurred. The reference is a scaled version of $r$ which is used to enable them to provide a specific level of voluntary effort. In this section, respresentative results from P1 are again shown to illustrate the various results which are examined in more detail in Section 4.2 .

\subsubsection{NV-LQT with no voluntary effort (NVO)}

First the LQT approach is investigated to examine baseline performance using the weighting parameters $Q=100$ and $R=1$. The first test does not consider voluntary effort in either model or experiment. It hence corresponds to traditional model-based control of FES. Here (25) was implemented to the controller with $f^{-1}(w, 0)$ and $f(u, 0)$ (i.e. no voluntary effort from participants). Representative tracking results are shown in Fig. 11. These show relatively accurate tracking, with a maximum amplitude close to that of the reference.

\subsection{2. $N V$-LQT with voluntary effort (NVI)}

The traditional model-based controllers are next applied in the realistic setting of rehabilitation where a patient applies voluntary effort. The controller was implemented as in the previous approach, but the participants were instructed to apply an 


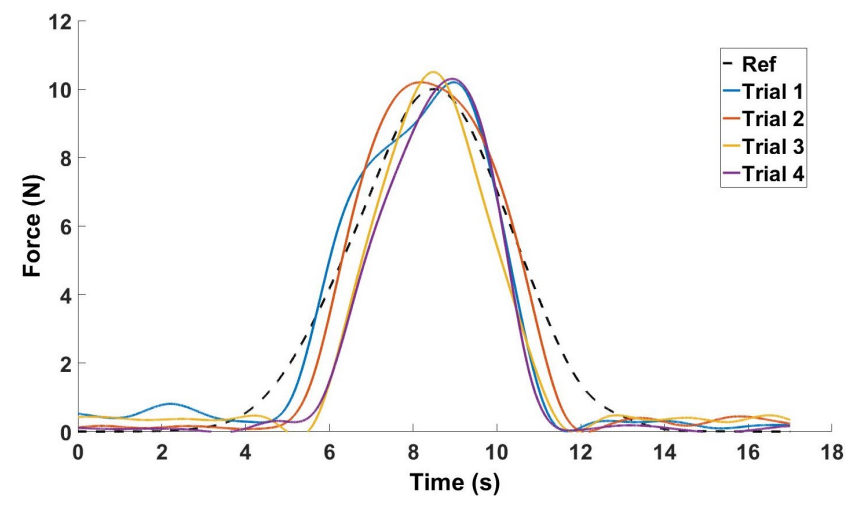

Figure 11: The output of LQT feedforward controller using NV model without voluntary effort

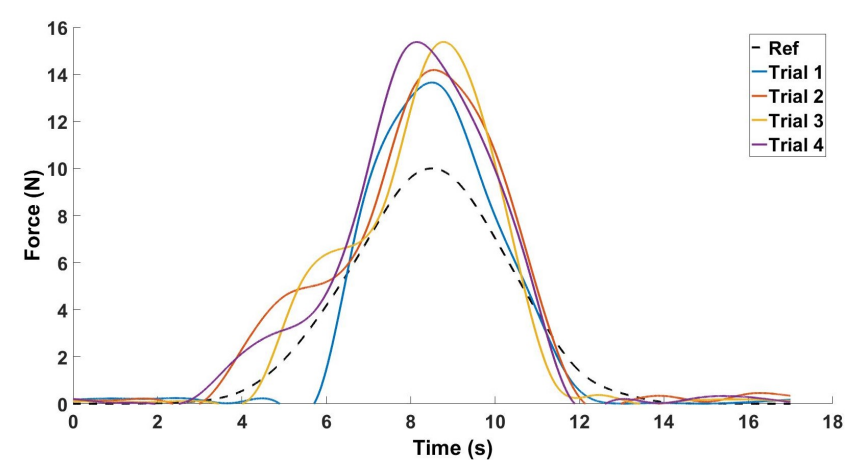

Figure 12: The output of LQT feedforward controller using NV model with voluntary effort

amount of voluntary effort. As stated, this was achieved by displaying a reference to them together with the EMG signal. The ${ }^{41}$ exact profile of voluntary contribution was arbitrary and determined by the participant. The maximum level typically corresponded to half that required to track the reference in order to mimic a recovering stroke participant. The results of this approach are illustrated in Fig. 12 The introduction of voluntary effort has caused significant overshoot.

\subsubsection{HA-LQT model with voluntary effort (HA)}

Next the new model-based control approach of Section 2 is applied. The models $f^{-1}(w, v)$ and $f(u, v)$ were used in the controller. The solution (25) was then applied with a small level of voluntary effort. Fig. 12 shows the tracking performance that has compensated for the presence of voluntary effort, removing the overshoot.

In the next subsections ILC is applied to examine whether the use of learning improves performance. Here 10 ILC trials are used, since this matches the clinical setting where patients must be highly engaged in completion of a variety of tasks with a limited time duration.

\subsubsection{NV-ILC with no voluntary effort (NVO)}

This approach does not consider voluntary intention. It is similar to Section 4.1.1, but the controller is now optimal ILC, and the weighting parameters are changed to $Q=5$ and $R=1$

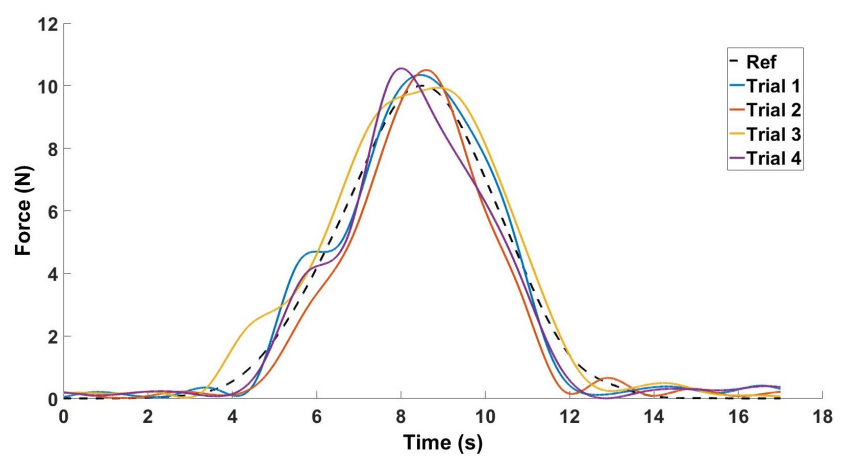

Figure 13: The output of LQT feedforward controller using HA model

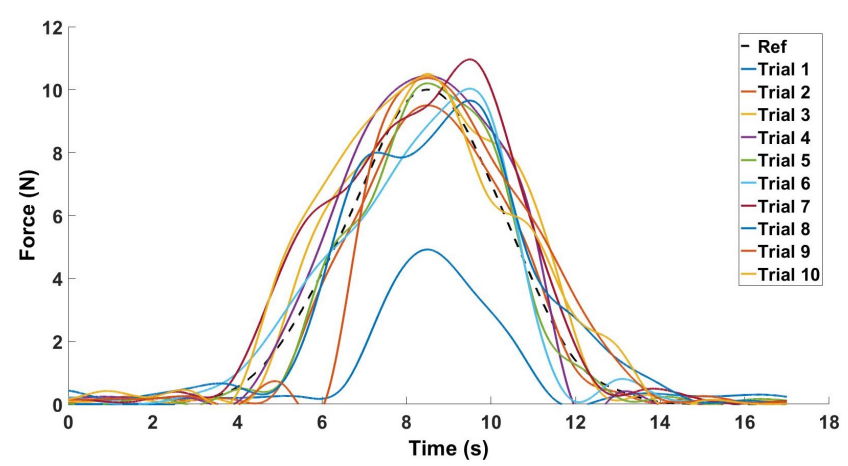

Figure 14: The output of ILC feedforward controller using NV model without voluntary effort

in order to better illustrate convergence. The solution (22) was applied with $v_{k}=0$. The participant does not apply voluntary effort. The tracking results are shown in Fig. 14

\subsubsection{NV-ILC with voluntary effort (NVI)}

The same ILC update is again applied, but with a level of voluntary effort provided by the participant (as in Section 4.1.2). The control input is given by 22 with $f^{-1}(w, 0)$ and $f(u, v)$. The results of this approach are shown in Fig. 15 and illustrate how traditional ILC deteriorates in the presence of voluntary effort.

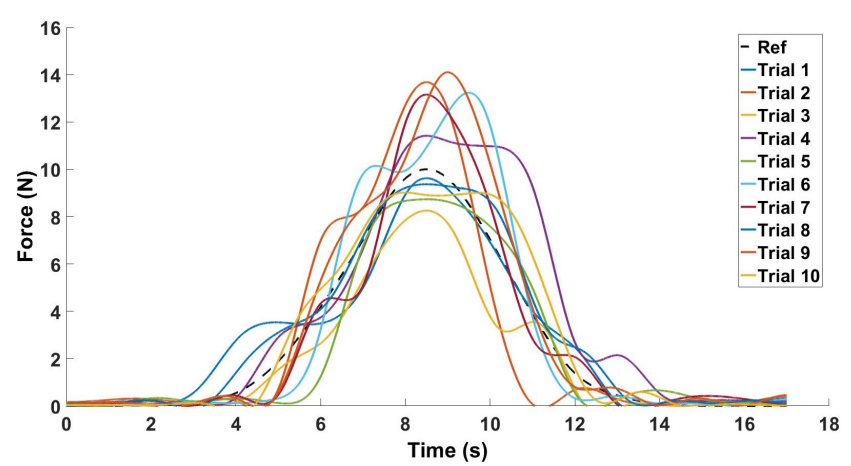

Figure 15: The output of ILC feedforward controller using NV model with voluntary effort 


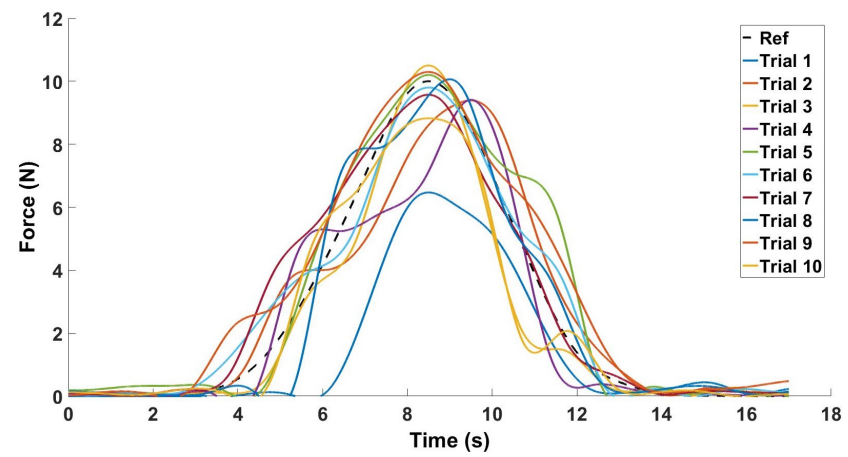

Figure 16: The output of ILC feedforward controller using HA model

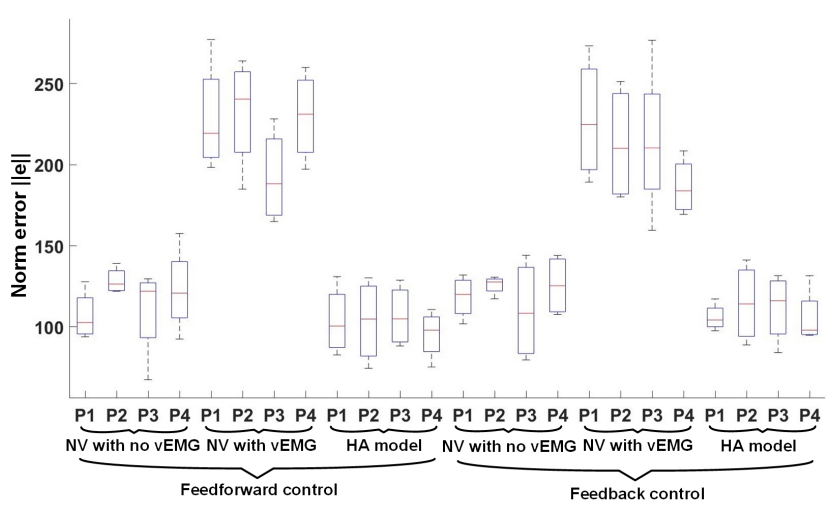

Figure 17: The error norm of participants using both feedforward, and feedback controller

\subsubsection{HA-ILC model with voluntary effort (HA)}

Next the hybrid model-based control approach of Section 2 is applied, using 22) with $u>0$ and $v>0$. Again the participant provides a small level of voluntary effort. Fig. 16 shows the tracking performance. This confirms that the new HA model can compensate for the presence of voluntary effort.

\subsection{Results}

The test procedure has been described for the feedforward implementation $(\sqrt{25})$ and $(22)$ ). It was then repeated for the feedback implementation (26) and (27)).

\subsection{1. $L Q T$}

The tests described in Section 4.1.1 to 4.1.3 were implemented with each participant. Fig. 17 shows the error norm of LQT using both NV and HA control structures for feedforward and feedback control schemes. These results show that the error norm of the NV form increased significantly when volitional effort is applied. However, the new HA model structures reduce it back to the same low levels. Table 1 illustrates the error norm of LQT using NV and HA forms for both controller schemes.

\subsubsection{ILC}

The tests described in Section 4.1.4 to 4.1.6 were implemented with each participant. The individual tracking results are shown in Fig. 18 to Fig. 21. Table 2 shows that the error norm of the traditional ILC structure with no voluntary effort

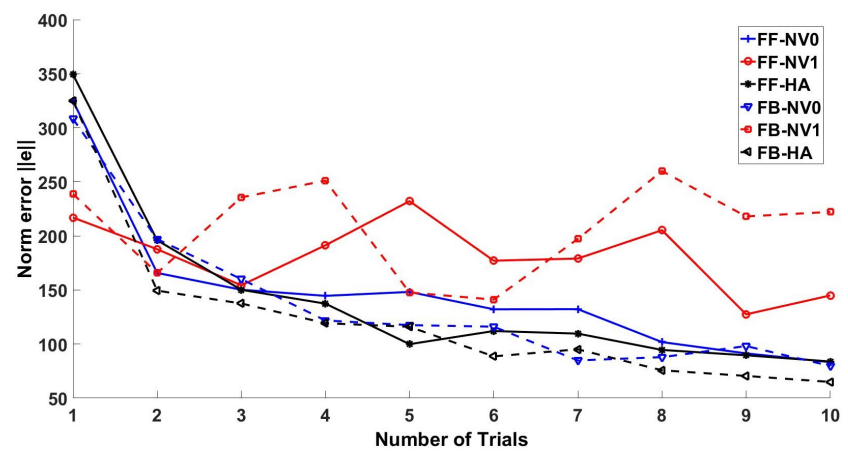

Figure 18: The error norm of P1 using optimal ILC feedforward, FF (dash line), and feedback control, FB (solid line)

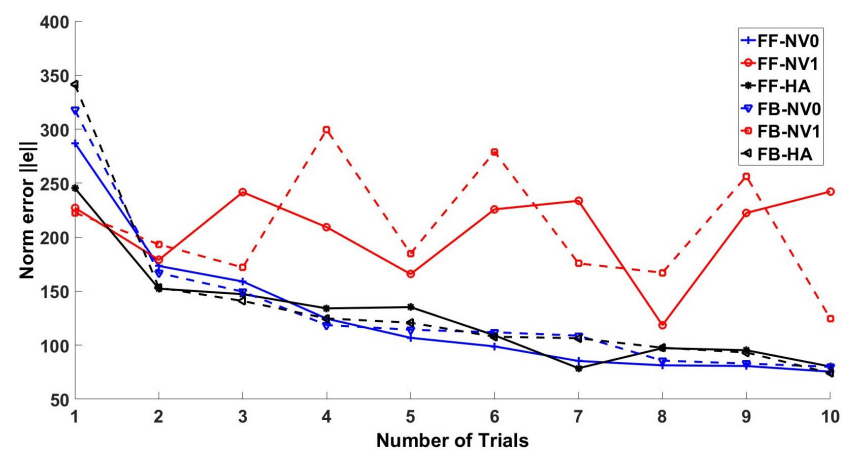

Figure 19: The error norm of P2 using optimal ILC feedforward, FF (dash line), and feedback control, FB (solid line)

supplied by the participant is similar to that of the new hybrid structure in the presence of voluntary intention. The results also show the substantial deterioration of traditional ILC in the presence of the same voluntary intention.

As previously stated, the first trial of ILC equates with LQT. ILC used a weight of $Q=5$, which means the first trial has a much greater error norm than the LQT results in Section 4.1.1 (which used $Q=100$ ). However, ILC quickly improves performance to provide superior levels of tracking.

The results using feedforward and feedback implementations are similar, but the feedforward control has greater error. The main advantage of the feedback implementation is computational efficiency because there is no need to calculate the large

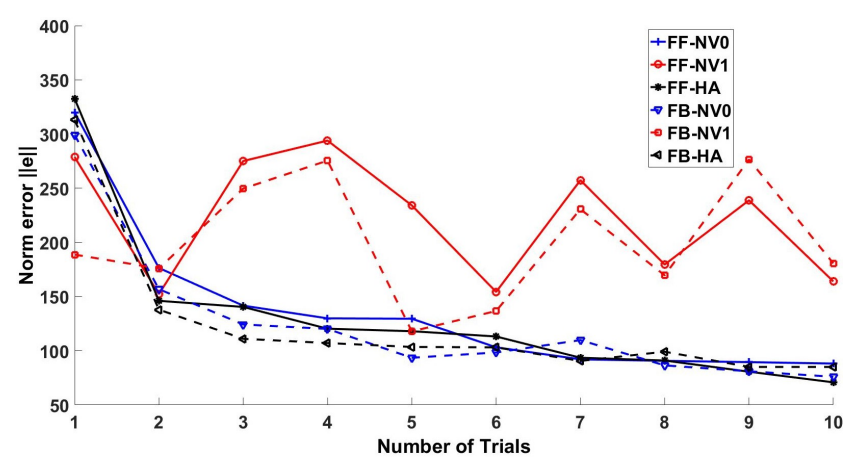

Figure 20: The error norm of P3 using optimal ILC feedforward, FF (dash line), and feedback control, FB (solid line) 
Table 1: The error norm of LQT feedforward and feedback control using NV and HA model, $(Q=100)$

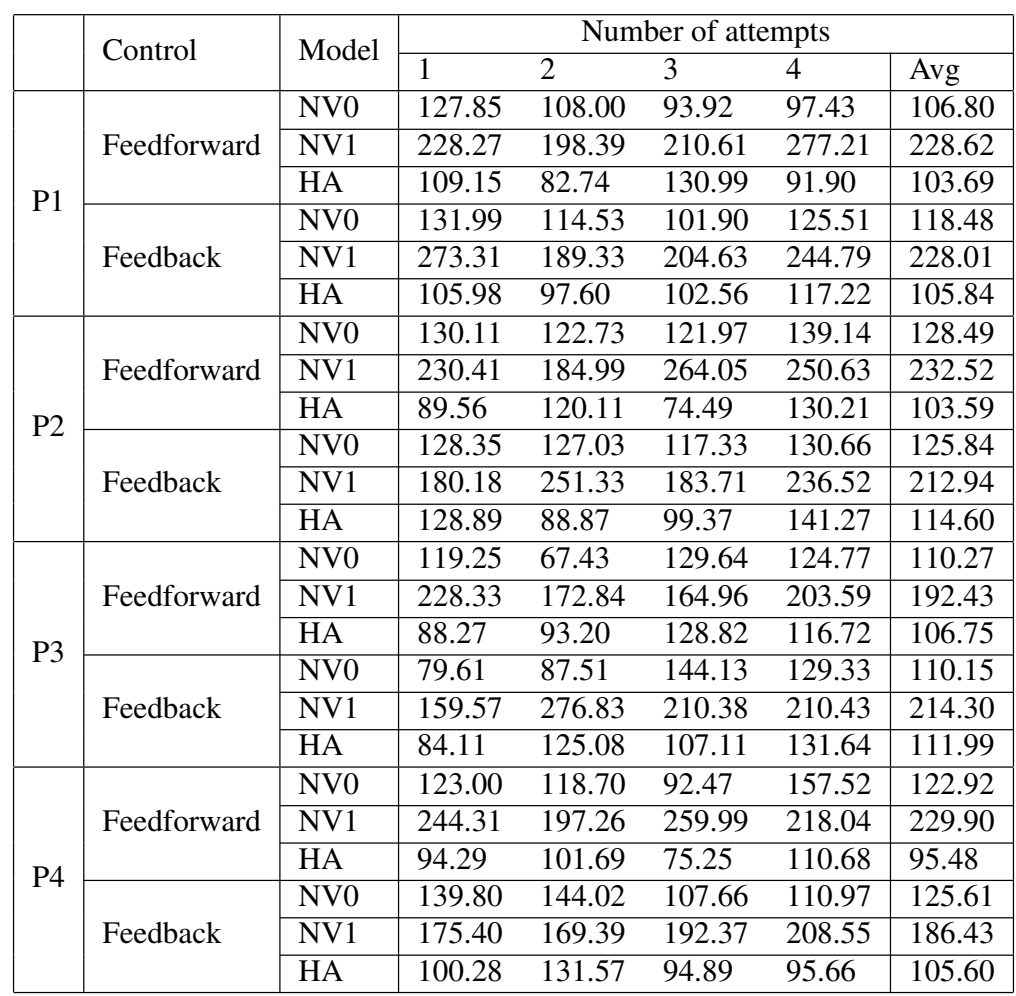

Table 2: The error norm of ILC feedforward and feedback control using NV and HA model, (Q=5)

\begin{tabular}{|c|c|c|c|c|c|c|c|c|c|c|c|c|c|}
\hline & \multirow{2}{*}{ Control } & \multirow{2}{*}{ Model } & \multicolumn{11}{|c|}{ Number of ILC trials } \\
\hline & & & 1 & 2 & 3 & 4 & 5 & 6 & 7 & 8 & 9 & 10 & Avg \\
\hline \multirow{6}{*}{$\mathrm{P} 1$} & \multirow{3}{*}{ Feedforward } & NV0 & 324.85 & 165.79 & 150.24 & 144.58 & 148.22 & 132.16 & 132.27 & 101.81 & 91.36 & 83.27 & 147.00 \\
\hline & & NV1 & 216.96 & 187.72 & 154.31 & 191.36 & 232.17 & 177.11 & 179.09 & 205.30 & 127.49 & 144.94 & 181.65 \\
\hline & & $\mathrm{HA}$ & 349.25 & 196.00 & 150.18 & 137.37 & 99.90 & 112.05 & 109.65 & 94.56 & 89.61 & 83.86 & 142.24 \\
\hline & \multirow{3}{*}{ Feedback } & NV0 & 308.30 & 197.08 & 160.12 & 121.81 & 117.52 & 116.07 & 84.88 & 87.89 & 98.16 & 79.78 & 137.16 \\
\hline & & NV1 & 238.75 & 165.76 & 235.66 & 251.17 & 147.66 & 141.14 & 197.26 & 259.99 & 218.04 & 222.23 & 207.77 \\
\hline & & HA & 324.88 & 149.42 & 137.52 & 119.29 & 115.95 & 88.61 & 95.14 & 75.73 & 70.51 & 64.95 & 124.20 \\
\hline \multirow{6}{*}{$\mathrm{P} 2$} & \multirow{3}{*}{ Feedforward } & NV0 & 287.07 & 173.33 & 158.93 & 124.50 & 106.78 & 98.85 & 85.35 & 81.35 & 80.69 & 75.45 & 127.23 \\
\hline & & NV1 & 227.14 & 178.98 & 241.64 & 209.24 & 165.80 & 225.75 & 233.63 & 118.50 & 222.42 & 242.27 & 206.54 \\
\hline & & HA & 245.34 & 152.32 & 147.21 & 134.07 & 135.22 & 109.10 & 78.67 & 97.23 & 95.32 & 80.20 & 127.47 \\
\hline & \multirow{3}{*}{ Feedback } & NV0 & 317.68 & 166.85 & 149.56 & 118.62 & 114.25 & 111.95 & 108.80 & 85.67 & 82.98 & 79.95 & 133.63 \\
\hline & & NV1 & 222.59 & 193.34 & 171.97 & 299.48 & 184.83 & 278.96 & 175.70 & 167.10 & 256.36 & 124.56 & 207.49 \\
\hline & & HA & 341.33 & 153.29 & 140.90 & 124.76 & 120.81 & 107.80 & 106.43 & 97.63 & 93.24 & 74.07 & 136.02 \\
\hline \multirow{5}{*}{ P3 } & \multirow{2}{*}{ Feedforward } & NV0 & 319.95 & 176.42 & 141.64 & 129.95 & 129.58 & 103.24 & 92.09 & 90.74 & 89.54 & 88.24 & 136.14 \\
\hline & & HA & 332.66 & 146.11 & 140.48 & 120.39 & 118.07 & 113.21 & 93.60 & 91.02 & 80.72 & 70.90 & 130.72 \\
\hline & \multirow{3}{*}{ Feedback } & NV0 & 299.21 & 156.87 & 124.31 & 120.20 & 93.58 & 98.47 & 109.87 & 86.53 & 81.10 & 76.02 & 124.62 \\
\hline & & NV1 & 188.73 & 175.93 & 249.71 & 275.49 & 117.92 & 136.74 & 230.67 & 169.64 & 276.53 & 180.71 & 200.21 \\
\hline & & HA & 312.98 & 137.97 & 111.08 & 107.16 & 103.57 & 103.17 & 90.67 & 99.18 & 85.11 & 85.10 & 123.60 \\
\hline \multirow{6}{*}{ P4 } & \multirow{3}{*}{ Feedforward } & NV0 & 237.72 & 152.64 & 148.39 & 131.75 & 115.42 & 100.89 & 94.03 & 73.75 & 83.89 & 75.75 & 121.42 \\
\hline & & NV1 & 191.88 & 264.37 & 142.14 & 263.18 & 193.60 & 158.45 & 139.06 & 175.66 & 224.34 & 169.13 & 192.18 \\
\hline & & HA & 265.96 & 157.23 & 149.46 & 119.46 & 112.86 & 118.94 & 102.55 & 89.36 & 78.36 & 71.81 & 126.60 \\
\hline & \multirow{3}{*}{ Feedback } & NV0 & 284.85 & 155.62 & 146.08 & 133.70 & 112.05 & 106.10 & 101.53 & 94.10 & 83.27 & 83.46 & 130.07 \\
\hline & & NV1 & 253.79 & 230.70 & 254.34 & 229.98 & 200.33 & 209.25 & 195.53 & 217.54 & 169.86 & 212.67 & 217.40 \\
\hline & & HA & 274.20 & 195.24 & 107.86 & 110.26 & 107.11 & 106.59 & 100.25 & 91.50 & 86.28 & 81.65 & 126.09 \\
\hline
\end{tabular}




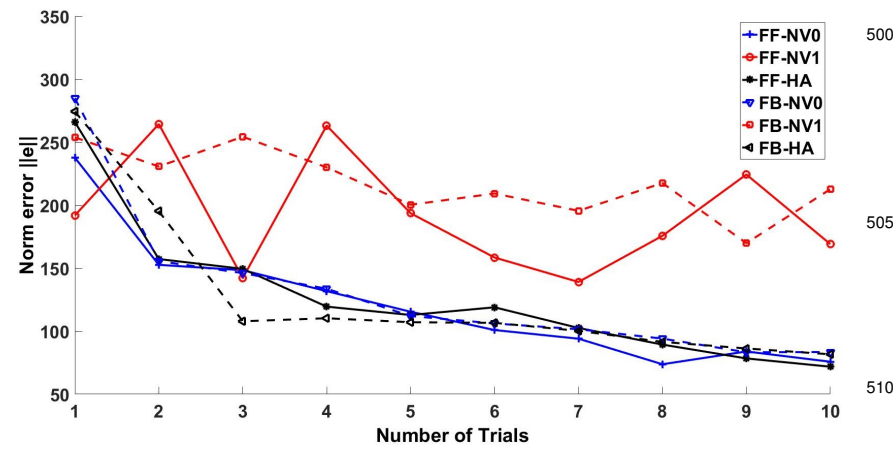

Figure 21: The error norm of P4 using optimal ILC feedforward, FF (dash line), and feedback control, FB (solid line)

$G$ matrix. However, this controller has a more complex structure to implement compared to the feedforward controller.

Due to the more complex hardware required, direct compar- ${ }^{520}$ ison with [6] is not possible. However, the results presented in [6] showed significant lag between output and reference, which 465 the current approach has addressed.

\section{Conclusion}

This paper has developed and evaluated a new hybrid model ${ }_{530}$ that incorporates voluntary effort, thereby enabling more sophisticated FES controllers to be developed that respond to a patient's residual movement capability. Since neurological recovery requires FES to both coincide with and maximise voluntary task contribution, this can be used to support more effective therapy. In addition, it has been validated in a clinicallyrelevant identification procedure. To illustrate its utility, the first model-based ILC scheme that uses both EMG and FES has been developed to provide motion control that balances accuracy and changes in voluntary muscle activation. Experimental results show that the controllers reduce the tracking error norm by between $20 \%$ and $40 \%$ in the case of each participant com- ${ }_{545}$ pared to traditional controllers based on models that neglect voluntary action. This confirms that the approach has significant potential to improve tracking, and is also beneficial for therapeutic purposes (since voluntary effort must be maximised $_{550}$ to promote effective rehabilitation). Tests were performed on unimpaired participants using conditions that mimic those of stroke patients who are recovering their voluntary action. These test conditions use similar, and indeed higher, levels of volun- ${ }_{555}$ tary contribution than could be anticipated, and therefore they are more demanding for the controller. Our testing procedure constitutes a necessary step before ethical approval for a clinical feasibility study with stroke participants.

Potential limitations are the time required for model identification and the identified model becoming less accurate due to effect such as fatigue. Future work will address this by extending the control framework to include multiple model adap-565 tive switching in order to eliminate the need for model identification. This will have a similar structure to [48] but with the plant model and controller augmented to include voluntary effort, and the inclusion of ILC following the lifted approach ${ }_{570}$ of [49]. This project will also be implemented experimentally with stroke participants.

[1] J. H. Burridge, M. Ladouceur, Clinical and therapeutic applications of neuromuscular stimulation: A review of current use and speculation into future developments, Neuromodulation 4 (4) (2001) 147-154.

[2] A. M. Hughes, J. H. Burridge, S. H. Demain, C. Ellis-Hill, C. Meagher, L. Tedesco-Triccas, R. Turk, I. Swain, Translation of evidence-based assistive technologies into stroke rehabilitation: users' perceptions of the barriers and opportunities, BMC Health Serv Res 14 (124) (2014) 1-12.

[3] O. A. Howlett, N. A. Lannin, L. Ada, C. Mckinstry, Functional electrical stimulation improves activity after stroke: A systematic review with metaanalysis, Arch. of Phys. Med. and Rehab. 96 (5) (2015) 934-943.

[4] C. Klauer, M. Irmer, T. Schauer, A muscle model for hybrid muscle activation, Current Direction in Biom. Eng. 1 (1) (2015) 386-389.

[5] T. Schauer, A. Vrontos, Modeling of mixed artificially and voluntary induced muscle contractions for controlled functional electrical stimulation of shoulder abduction, IFAC-PapersOnLine 51 (34) (2019) 284-289.

[6] C. Klauer, S. Ferrante, E. Ambrosini, U. Shiri, F. Dähne, I. Schmehl, A. Pedrocchi, T. Schauer, A patient-controlled functional electrical stimulation system for arm weight relief, Medical Eng. and Phys. 38 (11) (2016) 1232-1243.

[7] C. T. Freeman, A. M. Hughes, J. H. Burridge, P. H. Chappell, P. L. Lewin, E. Rogers, A model of the upper extremity using FES for stroke rehabilitation, Journal of Biomec. Eng. 131 (3) (2009) 1-12.

[8] T. Seel, C. Werner, J. Raisch, T. Schauer, Iterative learning control of a drop foot neuroprosthesis- Generating physiological foot motion in paretic gait by automatic feedback control, Cont. Eng. Prac. 48 (2016) 87-97.

[9] T. Seel, M. Valtin, C. Werner, T. Schauer, Multivariable control of foot motion during gait by peroneal nerve stimulation via two skin electrodes, IFAC-PapersOnLine 28 (20) (2015) 315-320.

[10] P. Müller, C. Balligand, T. Seel, T. Schauer, Iterative Learning Control and System Identification of the Antagonistic Knee Muscle Complex During Gait Using Functional Electrical Stimulation, IFAC-PapersOnLine 50 (1) (2017) 8786-8791.

[11] P. M. Aubin, M. S. Cowley, W. R. Ledoux, Gait simulation via a 6-DOF parallel robot with iterative learning control, IEEE Trans. on Biomed. Eng. 55 (3) (2008) 1237-1240.

[12] J. Bae, M. Tomizuka, A gait rehabilitation strategy inspired by an iterative learning algorithm, in: 18 th IFAC world congress, Vol. 44, Milano, Italy, (2008), pp. 2857-2864.

[13] V. Ghanbari, V. H. Duenas, P. J. Antsaklis, W. E. Dixon, Passivity-Based Iterative Learning Control for Cycling Induced by Functional Electrical Stimulation With Electric Motor Assistance, IEEE Trans. on Control Systems Tech. 27 (5) (2018) 2287-2294.

[14] M. Kutlu, C. Freeman, A. M. Hughes, M. Spraggs, A Home-based FES System for Upper-limb Stroke Rehabilitation with Iterative Learning Control, IFAC-PapersOnLine 50 (1) (2017) 12089-12094.

[15] F. Resquín, J. Gonzalez-Vargas, J. Ibáñez, F. Brunetti, J. L. Pons, Feedback error learning controller for functional electrical stimulation assistance in a hybrid robotic system for reaching rehabilitation, European J. of Trans. Myology 26 (3) (2016) 255-261.

[16] C. T. Freeman, Control system design for electrical stimulation in upper limb rehabilitation, Springer International Publishing, 2016.

[17] C. T. Freeman, A. M. Hughes, J. H. Burridge, P. H. Chappell, P. L. Lewin, E. Rogers, Iterative learning control of FES applied to the upper extremity for rehabilitation, Cont. Eng. Prac. 17 (3) (2009) 368-381.

[18] P. Sampson, C. T. Freeman, S. Coote, S. Demain, P. Feys, K. Meadmore, A. M. Hughes, Using functional electrical stimulation mediated by iterative learning control and robotics to improve arm movement for people with multiple sclerosis, IEEE Trans. on Neural Systems and Rehab. Eng. 24 (2) (2016) 235-248.

[19] A. Hughes, C. Freeman, J. Burridge, P. Chappell, P. Lewin, E. Rogers, Feasibility of iterative learning control mediated by functional electrical stimulation for reaching after stroke, Neurorehab. and Neural Repair 23 (6) (2009) 559-568.

[20] K. L. Meadmore, A. M. Hughes, C. T. Freeman, Z. Cai, D. Tong, J. H. Burridge, E. Rogers, Functional electrical stimulation mediated by iterative learning control and 3D robotics reduces motor impairment in chronic stroke, Journal of NeuroEng. and Rehab. 9 (1) (2012) 1-11.

[21] S. Sa-e, C. T. Freeman, K. Yang, Model-based control of FES embed- 
ding simultaneous volitional EMG Measurement, in: 12th UKACC IEEE Conf. on Control., Sheffield, UK, (2018), pp. 480-485.

[22] A. F. Huxley, Muscle structure and theories of contraction., Progress in biophysics and biophysical chemistry 7 (1957) 255-318. Proceeding of the Royal Society (126) (1938) 136-195.

[24] M. L. Audu, D. T. Davy, The influence of muscle model complexity in musculoskeletal motion modeling, Journal of Biomec. Eng. 107 (2) (1985) 147-157.

[25] R. Flaherty, C. Robinson, G. Agarwal, Determining appropriate models for joint control using surface electrical stimulation of soleus in spinal cord injury, Med. Biol. Eng. Comput. 32 (1994) 273-282.

[26] J. M. Winters, Hill based muscle model: A systems engineering perspective, in Multiple Muscle System (1990) 69-93

[27] S. J. Dorgan, M. J. O'Malley, A nonlinear mathematical model of electrically stimulated skeletal muscle, IEEE Trans. on Rehab. Eng. 5 (2) (1997) 179-194.

[28] S. Jenzernik, R. V. Wassink, T. Keller, Sliding mode closed-loop control of FES: Controlling the shank movement, IEEE Trans. on Biom. Eng. 51 (2) (2004) 263-272.

[29] N. Lan, Stability analysis for postural control in a two-joint limb system, IEEE Trans on Neural Systems and Rehab. Eng. 10 (4) (2002) 249-259.

[30] R. Riener, T. Fuhr, Patient-driven control of FES-supported standing up: a simulation study, IEEE Trans. on Rehab. Eng. 6 (2) (1998) 113-124.

[31] T. Schauer, N. O. Negård, F. Previdi, K. J. Hunt, M. H. Fraser, E. Ferchland, J. Raisch, Online identification and nonlinear control of the electrically stimulated quadriceps muscle, Cont. Eng. Prac. 13 (9) (2005) 12071219.

[32] C. Lynch, M. Popovic, D. Rushton, Functional electrical stimulation: Closed-loop control of induced muscle contractions, IEEE Control Systems Magazine 28 (2) (2008) 40-50.

[33] M. Ferrarin, F. Palazzo, R. Riener, J. Quintern, Model-based control of FES-induced single joint movements, IEEE Trans. on Neural Systems and Rehab. Eng. 9 (3) (2001) 245-257.

[34] E. J. Dempsey, D. T. Westwick, Identification of hammerstein models with cubic spline nonlinearities, IEEE Trans. on Biomed. Eng. 51 (2) (2004) 237-245.

[35] K. J. Hunt, M. Munih, N. N. De Donaldson, F. M. Barr, Investigation of the Hammerstein hypothesis in the modeling of electrically stimulated muscle, IEEE Trans. on Biom. Eng. 45 (8) (1998) 998-1009.

[36] R. Baratta, M. Solomonow, The dynamic response of nine different skeletal muscles, IEEE Trans. on Biom. Eng. 37 (3) (1990) 243-251.

[37] W. K. Durfee, K. E. MaClean, Methods for estimating isometric recruitment curves of electrically stimulated muscle, IEEE Trans. on Biom. Eng. 36 (7) (1989) 654-667.

[38] J. Bobet, E. R. Gossen, R. B. Stein, A comparison of models of force production during stimulated isometric ankle dorsiflexion in humans, IEEE Trans. on Neural Systems and Rehab. Eng. 13 (4) (2005) 444-451.

[39] E. Langzam, E. Isakov, J. Mizrahi, Evaluation of methods for extraction of the volitional EMG in dynamic hybrid muscle activation., Journal of neuroeng. and rehab. 3 (27) (2006) 1-11.

[40] L. A. Bernotas, , P. E. Crago, H. J. Chizeck, A discrete-time model of electrically stimulated muscle, IEEE Trans on Biomed. Eng. 33 (9) (1986) 829-838.

[41] F. Le, I. Markovsky, C. T. Freeman, E. Rogers, Identification of electrically stimulated muscle models of stroke patients, Cont. Eng. Prac. 18 (4) (2010) 396-407.

[42] E. H. Copur, C. T. Freeman, B. Chu, D. S. Laila, System identification for FES-based tremor suppression, European Journal of Control 27 (2016) 45-59.

[43] D. A. Bristow, M. Tharayil, A. G. Alleyne, A survey Of iterative learning control: A learning-based method for high-performance tracking control, IEEE Control Systems 26 (3) (2006) 96-114.

[44] E. Rogers, D. H. Owens, H. Werner, C. T. Freeman, P. L. Lewin, S. Kichhoff, C. Schmidt, G. Lichtenberg, Norm-optimal iterative learning control with application to problems in accelerator-based free electron lasers and rehabilitation robotics, Euro. J. of Control 16 (5) (2010) 497-522.

[45] N. Amann, D. Owens, Iterative learning control for discrete-time systems with exponential rate of convergence, IEE Proceedings - Control Theory and Applications 143 (2) (1996) 217-224.

[46] Y. Muraoka, Development of an EMG recording device from stimulation electrodes for functional electrical stimulation., Front. Med. Biol. Eng. 11 (4) (2002) 323-333.

[47] W. K. Durfee, K. I. Palmer, Estimation of force-activation, force-Length, and force-velocity properties in isolated, electrically stimulated muscle, IEEE Trans. on Biom. Eng. 41 (3) (1994) 205-216.

[48] O. Brend, C. T. Freeman, M. French, Multiple-model adaptive control of functional electrical stimulation, IEEE Trans. on Cont. Systems Tech. 23 (5) (2015) 1901-1913.

[49] C. T. Freeman, Multiple model switched repetitive control, in: 56th IEEE Conf.on Deci. and Cont., Melbourne, Australia, (2017), pp. 4801-4806. 